\title{
International
}

\section{Scope and contributions}

International Psychogeriatrics is written by and for those doing clinical, teaching, and research work with older people. It is the official journal of the International Psychogeriatric Association (IPA) and is published by Cambridge University Press, Cambridge, UK. Although it is primarily concerned with psychogeriatrics, the journal welcomes contributions from all concerned with the field of mental health and aging. Original research papers are particularly sought.

Contributions include original research articles, case reports, reviews of the literature, book reviews, letters to the editor, and editorials. Apart from editorials and book reviews, which are commissioned, contributions to International Psychogeriatrics are spontaneously written and submitted by authors. Papers are reviewed by two expert reviewers selected by the Editor-in-Chief. At present, about half of the papers submitted are accepted for publication. The journal's Science Citation Index impact factor is 1.89 (2013). Submission of a paper implies that it is neither under consideration for publication elsewhere, nor previously published in English. Manuscripts must be formatted double-spaced with ample margins on all sides and the pages should be numbered. International Psychogeriatrics uses the spelling of American English. Manuscripts written by those whose primary language is not English should be edited carefully for language prior to submission. The journal has a Language Assistance Panel consisting of both native English speakers willing to check mauscripts for style prior to submission, and bilingual experts willing to assist with the translation of manuscripts into English. Further details including contact information for individual panel members can be found at both the journal and IPA websites (journals.cambridge.org/ipg and www.ipa-online.org).

An up to date version of the journal's instructions for contributors can be found at the International Psychogeriatrics website journals. cambridge.org/ipg. Please read these instructions carefully before submitting articles. Articles that are not prepared in accordance with these guidelines will be returned to authors.

Manuscripts should be submitted online via our manuscript submission and tracking site, <http://mc.manuscriptcentral.com/ipg >. Full instructions for electronic submission are available directly from this site.

\section{Important Addresses}

Office of the Editor-in-Chief

NicolaT. Lautenschlager Editor-in-Chief,

International Psychogeriatrics

RMH Royal Park Campus

34-54 Poplar Road,

Parkville,Victoria 3052

Australia

Email:ipaj-ed@unimelb.edu.au

$\mathrm{Tel}+61398160485$

Fax +61398160477

\section{For business matters:}

Ms. Susan M. Oster

Managing Editor, International Psychogeriatrics

International Psychogeriatric Association

550 Frontage Road,

Suite 3759

Northfield, IL 60093

USA

Email:ipa@ipa-online.org

Tel: +18475013310

Fax: +1 8475013317

Web: www.ipa-online.org

\section{For book review matters:}

Professor David Ames

Normanby House

St. George's Hospital

283 Cotham Road

Kew, Victoria, 3101

Australia

Email:

dames@unimelb.edu.au

Tel: +61419378455

FAX: +61398160477

This journal issue has been printed on FSC-certified paper and cover board. FSC is an independent, non-governmental, not-for-profit organization established to promote the responsible management of the world's forests. Please see www.fsc.org for information.

Enquiries about advertising should be sent to the Journal's Promotion Department of the Cambridge or American Branch of Cambridge University Press. 


\section{International Psychogeriatrics}

\section{CONTENTS}

Guest Editorial 1411 Mental health issues and discrimination among older LGBTI people

Jean Tinney, Briony Dow, Phillip Maude, Rachel Purchase, Carolyn Whyte and Catherine Barrett (Australia)

Commentary 1417 A useful development in measuring activities of daily living

David Ames (Australia)

Paper of the Month 1419 Discriminative power of the advanced activities of daily living (a-ADL) tool in the diagnosis of mild cognitive impairment in an older population P. De Vriendt, T. Mets, M. Petrovic and E. Gorus (Belgium)

Reviews 1429 Use of CSF $\alpha$-synuclein in the differential diagnosis between Alzheimer's disease and other neurodegenerative disorders Zheng-Yu Wang, Zhen-Min Han, Qi-Fei Liu, Wei Tang, Kui Ye and Yu-You Yao (China)

1439 A systematic narrative review of support groups for people with dementia G. R. Toms, L. Clare, J. Nixon and C. Quinn (UK)

Original Research 1467 No lower cognitive functioning in older adults with attention-deficit/hyperactivity disorder E. J. Semeijn, N. C. M. Korten, H. C. Comijs, M. Michielsen, D. J. H. Deeg, A. T. F. Beekman and J. J. S. Kooij (the Netherlands)

1477 No evidence in favor of a more deleterious impact of a major depressive episode on verbal memory in older patients with antidepressant response Elise Blandin, Guilhem Carle, Benoit Theuil, Julien Katz and Philip Gorwood (France)

1485 In depressed older persons higher blood pressure is associated with symptoms of apathy. The NESDO study J. E. F. Moonen, A. J. M. de Craen, H. C. Comijs, P. Naarding, W. de Ruijter and R. C. van der Mast (the Netherlands)

1495 Validity of the geriatric depression scale and the collateral source version of the geriatric depression scale in nursing homes Zhicheng Li, Yun-Hee Jeon, Lee-Fay Low, Lynn Chenoweth, Daniel W. O'Connor, Elizabeth Beattie and Henry Brodaty (Australia)

1505 Development and validation of the geriatric depression inventory in Chinese culture Zhijuan Xie, Xiaozhen Lv, Yongdong Hu, Wanxin Ma, Hengge Xie, Kai Lin, Xin Yu and Huali Wang (China)

1513 Advancing the screening of fibromyalgia in late-life depression: practical implications for psychiatric settings John R. Jochum, Amy E. Begley, Mary Amanda Dew, Debra K. Weiner and Jordan F. Karp (USA)

1523 Differences in anxiety and depression symptoms: comparison between older and younger clinical samples Viviana M. Wuthrich, Carly J. Johnco (Australia) and Julie L. Wetherell (USA)

1533 Validation of the geriatric anxiety inventory in a duloxetine clinical trial for elderly adults with generalized anxiety disorder Susan G. Ball, Sarah Lipsius and Rodrigo Escobar (USA)

1541 Ageist attitudes block young adults' ability for compassion toward incapacitated older adults Yoav S. Bergman and Ehud Bodner (Israel)

1551 An evaluation of palliative care contents in national dementia strategies in reference to the European Association for Palliative Care white paper Miharu Nakanishi, (Japan) Taeko Nakashima, (USA) Yumi Shindo, Yuki Miyamoto, (Japan) Dianne Gove, (Luxembourg) Lukas Radbruch (Germany) and Jenny T. van der Steen (the Netherlands)

1563 The economic impact of moderate stage Alzheimer's disease in Italy: evidence from the UP-TECH randomized trial Carlos Chiatti, Gianluca Furneri, Joseph M. Rimland, Federica Demma, Franco Bonfranceschi, Laura Cassetta, Filippo Masera, Antonio Cherubini, Andrea Corsonello and Fabrizia Lattanzio, on behalf of the UP-TECH research group (Italy)

Case Report 1573 Capgras syndrome in a very late onset, treatment resistant schizophrenia M. K. Nurul Ain, R. Rosdinom and M. Raynuha (Malaysia)

Letter 1577 Comparing the Performance of the HADS and the GDS-15 in the AIBL Study

Guy Campbell, Christina Bryant, Kathryn A. Ellis, Rachel Buckley, David Ames and The Aibl Research Group (Australia) 